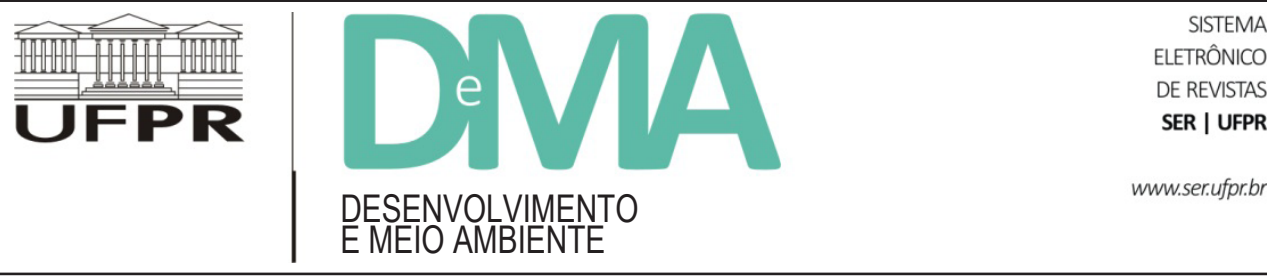

\title{
Estudios de percepción ambiental: aproximaciones hacia la filosofía y práctica del yoga como herramientas hermenéuticas
}

\section{Estudos de percepção ambiental: aproximações da filosofia e da prática da yoga como ferramentas hermenêuticas}

\section{Environmental perception studies: approaches to the philosophy and practice of yoga as hermeneutical tools}

\author{
Victoria D'HERS $S^{1,2,3^{*}}$ \\ ${ }^{1}$ Instituto de Investigaciones Gino Germani (IIGG), Facultad de Ciencias Sociales, Universidad de Buenos Aires, Argentina. \\ ${ }^{2}$ Consejo Nacional de Investigaciones Científicas y Técnicas (CONICET), Buenos Aires, Argentina. \\ ${ }^{3}$ Centro de Investigaciones y Estudios Sociológicos (CIES), Buenos Aires, Argentina. \\ *E-mail de contacto: victoriadhers@gmail.com
}

Artículo recibido el 11 de noviembre, 2018, versión final aceptada el 30 de mayo, 2019.

RESUMEN: En el presente escrito desplegamos los avances de una indagación ligada a la percepción ambiental y las sensibilidades sociales. La pregunta que enmarca la investigación pone el foco en comprender los modos sociales de configuración de las sensibilidades, siendo estas constitutivas de la percepción. En particular, tratamos aquí lo referente a la práctica del yoga en tanto una técnica corporal cada vez más extendida en las sociedades contemporáneas, observando de qué modos esta práctica genera cierta percepción específica, tanto del cuerpo propio como del ambiente, con miras a complejizar el entendimiento de la problemática del "ambiente", la percepción, y la conciencia para observar de qué manera generan, necesariamente, vínculos con el "entorno". Como trasfondo está la pregunta por la misma idea de límites y bordes entre el cuerpo propio, el ambiente, y sus mutuas configuraciones. Aspirando a sumar a la construcción de una perspectiva que parta desde la experiencia del sur global es que se revisan los fundamentos de ambos términos de la problemática, desde una mirada crítica de la modernidad colonial.

Palabras clave: ambiente; cuerpo/emoción; yoga; percepción. 
RESUMO: No presente escrito apresentamos os avanços de uma investigação ligada à percepção ambiental e às sensibilidades sociais. A pergunta que enquadra a pesquisa coloca o foco na compreensão dos modos sociais de configuração das sensibilidades, sendo estes constitutivos da percepção. Em particular, tratamos sobre a prática do yoga como uma técnica corporal cada vez mais difundida nas sociedades contemporâneas, observando de que maneiras cria certa percepção específica, tanto do próprio corpo quanto do ambiente, para aprofundar o entendimento das problemáticas do "ambiente", da percepção e da consciência, para observar como eles criam, necessariamente, ligações "ambientais". Com isso, questionamos os limites e as fronteiras entre o próprio corpo, o ambiente e suas configurações mútuas. Este trabalho aspira se unir à construção de uma experiência do sul global com base nos fundamentos de ambos os termos da questão, revistos desde uma perspectiva crítica da modernidade colonial.

Palavras-chave: ambiente corpo/emoção; yoga; percepção.

ABSTRACT: In these pages we display some headings of a research linked to environmental perception and social sensibilities. The aim is to understand the social modes of configuration of sensibilities, being constituents of perception. Particularly, we deal here with some considerations of yoga bodily practices increasingly extended in urban societies, observing in which ways this generates a specific perception, both of the body (flesh) and the environment, so as to amplify our views of the "green" issue, the perception and consciousness, in order to observe how they connect to the "surroundings". As a background, there is the question of the limits and boundaries between body, environment and their mutual configurations. Aiming at constructing a perspective that goes from global south, we revise these from a critical view of a colonised modernity.

Keywords: environment; body/emotion; yoga; perception.

\section{Introducción}

En el presente escrito desplegamos los avances de una indagación ligada a la percepción ambiental y las sensibilidades sociales. ${ }^{1}$ La pregunta que enmarca la investigación pone el foco en comprender los modos sociales de configuración de las sensibilidades, siendo estas constitutivas de la percepción.
En particular, tratamos aquí lo referente a la práctica del yoga en tanto una técnica corporal cada vez más extendida en las sociedades contemporáneas, observando de qué modos esta práctica genera cierta percepción específica, tanto del cuerpo propio como del ambiente.

Cabe destacar que dentro de una sociología de los cuerpos/emociones, entendemos que ambos términos conforman una totalidad, separada solo

\footnotetext{
${ }^{1}$ El plan de trabajo financiado por el Consejo Nacional de Investigaciones Científicas y Técnicas CONICET, con Lugar de Trabajo en el Instituto de Investigaciones Gino Germani, Universidad de Buenos Aires. Para el periodo 2018-19, se titula "Construcción de las sensibilidades, percepción y política de los cuerpos. Análisis e interpretación de(sde) la expresividad." El Objetivo General es el de comprender las relaciones entre percepción, sensibilidad y contaminación ambiental en sus conexiones con las formas de construcción social de las sensibilidades, en condiciones de habitabilidad precaria. Los Objetivos Específicos se detallan a continuación: 1. Explicitar los esquemas de percepción y acción referentes a la contaminación ambiental de sujetos en condiciones de habitabilidad precaria. 2. Analizar las sensibilidades relacionadas con el ambiente y construcción de la espacialidad, y las definiciones en torno a la "contaminación" de sujetos en condiciones de habitabilidad precaria. 3. Establecer las relaciones entre el habitus de clase, acostumbramiento y percepción de la contaminación ambiental en sujetos en condiciones de habitabilidad precaria. 4. Profundizar la aplicabilidad de técnicas de autoconocimiento, en las formas de interpretación del material surgido de las metodologías expresivo-creativas para explicitar relaciones de la percepción y sensibilidad, esquemas de acción y la contaminación de sujetos en condiciones de habitabilidad precaria. 5. Indagar las sensibilidades sociales ligadas a las relocalizaciones vinculadas con la contaminación ambiental.
} 
analíticamente. Pero justamente en la tarea de comprenderla, es donde debemos reelaborar las conexiones y desconexiones que operan en y entre ambos niveles. En este sentido, según investigaciones precedentes ligadas al ambiente y la contaminación, se ha observado que el ambiente (entendido, en primera instancia, como el espacio exterior, donde el límite se delinea entre la casa propia y el afuera) está invisibilizado, siendo esto parte de una determinada política de los cuerpos/emociones.

Es así que, con miras a complejizar el entendimiento de la problemática del "ambiente" y la percepción ambiental, hemos iniciado un abordaje de ciertas técnicas corporales para observar de qué modos generan, necesariamente, vínculos con el "entorno". Asimismo, indican la necesidad de este trabajo y revisiones teórico- epistemológicas, lo que algunos autores refieren como crisis de la sociología ligada a la percepción ambiental. ${ }^{2}$

Como trasfondo está la pregunta por la misma idea de límites y bordes entre el cuerpo propio, el ambiente, y sus mutuas configuraciones. Aspirando a sumar a la construcción de una perspectiva que parta desde la experiencia del sur global, es que se revisan los fundamentos de ambos términos de la problemática. Por un lado, el lugar de enunciación propio del pensamiento ambiental latinoamericano, haciéndonos eco de la idea de que "es desde la radicalidad epistemológica del concepto de ambiente
- que nace de la crisis ambiental como punto límite de la racionalidad dominante - de donde surge un saber ambiental emancipador, arraigado en los potenciales ecológicos y en la creatividad cultural de los territorios del sur" (Leff, 2017, p. 145).

Por otro, la revisión de los límites y potencialidades de esa apropiación epistemológica partiendo de otros paradigmas, como lo es en este caso del sostenido desde la filosofía milenaria del yoga. Finalmente, se aspira a conectar preliminarmente ambos términos a través de la forma que son socialmente configurados en la actualidad.

Cabe aclarar que han existido varios intentos de acercamientos entre los llamados paradigmas oriental-occidental. Autores como Ken Wilber han ahondando en estas vinculaciones, y las posibles confusiones a las que paralelismos apresurados pueden llevar. ${ }^{3}$ Sabiendo que la física y la mística se mueven por carriles diversos, a nivel cualitativo de que cada nivel de conocimiento implica un salto creativo, algo emergente; a la vez también hay ejemplos de encuentros potentes entre ambos. Entonces, si bien no trasladamos afirmaciones del campo espiritual hacia el estudio científico, sí vemos potencialidad en dejar que algunas afirmaciones sean revisadas desde otros campos del conocimiento. $\mathrm{Pa}$ rafraseando a Albert Einstein, la intención del arte y la ciencia, en última instancia, responde a una misma motivación: navegar en el Misterio de lo sublime. ${ }^{4}$

\footnotetext{
2 "La dispersión y la pobre concreción de los estudios, la falta de imaginación teórica y metodológica, las dificultades para encontrar explicaciones satisfactorias a determinados problemas presentados en la evidencia empírica o la lentitud para integrar los avances de los diferentes campos e investigaciones son algunos de los síntomas del atasco que, a juicio de los autores mencionados, afecta a la sociología de la percepción ambiental" (Cerrillo Vidal, 2010, p. 34).

3 "Es verdad que el intento actual de aislar ciertos puntos comunes a todas estas fuerzas [físicas, biológicas, psicológicas] es legítimo, pero ya podemos comprender lo sumamente cuidadosos que hay que ser en ese empeño.” (Wilber, 1986, p. 52).

${ }^{4}$ Excedería estas páginas abrir la discusión acerca de las vinculaciones entre ciencia y religión. Es destacable, sin embargo, que hay científicos que han planteado este tema, afirmando que hay una unidad última que vincula todas las diversas fuerzas, más en el sentido de un "sentimiento religioso cósmico", antes que una religión basada en preceptos morales, mono o politeístas, etcétera: "El 'sentimiento religioso cósmico' surge de la constatación de la impotencia del ser humano ante la Naturaleza, ante el orden sublime y maravilloso que en ella se revela y ante el mundo de las ideas que respecto de ella nos creamos. La racionalidad o inteligibilidad del mundo es una convicción cercana a la convicción religiosa" (Daros, 2002, p. 47, sobre Einstein).
} 
Es así que, dentro de los intereses de este escrito, la pregunta por cómo el dominio técnico-corporal presenta potencialidades para generar diversas vinculaciones con el ambiente, es transversal. Es necesario repensar, luego, cómo la noción de ambiente será un modo de abrir conexiones epistemológicas con el concepto de cuerpo.

El lector podrá preguntarse del por qué de este cruce. En primer término, se basa en un recorrido vital. Quien escribe, como parte de las clases medias urbanas de una gran ciudad como Buenos Aires, se ha encontrado con esta práctica/disciplina hace veinte años (véase D’hers, 2018a). Pero más allá de la propia narrativa de caminos de indagación que se sintetizan y confluyen a lo largo de una experiencia de vida, este atravesamiento cobra sentido en tanto entendido también como parte del ahora definido "sur global". 5

Por una parte, el sur global como objeto de un avance colonial perpetuo, lo que (nos) obliga a re-posicionarnos en medio de la resignificación de culturas y prácticas milenarias. Según hemos visto en otro lado (D'hers, 2018b), esta práctica en particular está cada vez más extendida a nivel urbano, al mismo tiempo que se emparenta con un modo de consumo y acumulación de experiencias, entrando asimismo en una matriz de "rendimiento" propia de la cultura occidental moderna.

Por otra, las potencialidades de re-subjetivación asumiendo cierta posición en tanto culturas urbanas atravesadas por múltiples confluencias que pueden, o bien ser un modo más de consumo, o devenir destellos hacia otros modos de entendimiento del ser, y del ser en tanto parte de algo mayor. Parti- cularmente aquí, entonces, analizamos esto en tanto vinculaciones posibles con el ambiente, en un siglo marcado por la depredación de energías en término amplio (naturales, que implican las “corporales").

Finalmente, cabe remarcar que no revisamos la práctica del yoga en términos sociodemográficos, o en cuanto a su impacto o alcances, etcétera; sino que, en esta primera aproximación, observamos lo referente a la filosofía que presenta y sus posibilidades cognitivo/afectivas, hacia una mirada crítica de nuestra comprensión de la conciencia. Queda, para futuras indagaciones, realizar el análisis de la experiencia y del estar-ahí, de quienes practican este tipo de disciplinas.

Anima estas reflexiones la sospecha de que, así como la depredación se alimenta de territorios, cuerpos y sensibilidades, cualquier alternativa a la crisis civilizatoria actual nace también de una reapropiación y direccionamiento de la energía vital disponible, clave del funcionamiento del metabolismo personal y social, en sentido amplio.

El trabajo se organiza como sigue: se recorren ciertas implicancias del concepto naturaleza, para delinear la idea de ambiente como síntesis epistemológica más abarcadora, abriendo la posibilidad de, en el segundo apartado (dividido en dos), recorrer perspectivas sobre el cuerpo. En una primera parte, delineamos lo que entendemos que es el proceso de percepción como actividad situada, retomando una mirada constructivista, revisando cómo opera el borde, no como límite sino en tanto diferenciación dinámica. En la segunda, aportamos a una breve revisión del estado del arte de los estudios del yoga en general y como vinculación con la

\footnotetext{
5 "El Sur global no es entonces un concepto geográfico, aún cuando la gran mayoría de estas poblaciones viven en países del hemisferio Sur. Es más bien una metáfora del sufrimiento humano causado por el capitalismo y el colonialismo a escala global y de la resistencia para superarlo o minimizarlo. Es por eso un Sur anticapitalista, anticolonial y antiimperialista. Es un Sur que existe también en el Norte global, en la forma de poblaciones excluidas, silenciadas, marginadas, como lo son los inmigrantes sin papeles, los desempleados, las minorías étnicas o religiosas, las víctimas del sexismo, de homofobia y de racismo" (de Sousa Santos, 2010, p. 49).
} 
percepción en tanto proceso. Finalmente, presentamos reflexiones sobre las implicancias de la noción de unión y cómo podemos nutrir lo dicho hasta aquí desde otro abordaje y matriz epistemológica.

\section{Naturaleza como recurso vs. ambiente como totalidad}

Mucho se ha debatido en torno a la noción de naturaleza, y sus vinculaciones con la cultura. La historia misma de la antropología, por ejemplo, nos sitúa en esa discusión. Antes que hacer un recorrido exhaustivo por dicho debate, aspiramos aquí a sentar las bases de lo que entendemos que pueda guiarnos a comprender la unión, antes que la escisión (disciplinar, existencial) entre términos como naturaleza, entorno, energía. Esto con miras a visibilizar la trama existente entre los modos como comprendemos "lo natural", y las vinculaciones con el cuerpo y la sensibilidad que derivan de dicha comprensión. Es desde esta revisión que abordamos la tensión entre la idea de naturaleza y ambiente, para hilvanarnos en una totalidad indivisible del "entorno", que puede ser la puerta de entrada para unificar ciertos análisis y abordajes de las sensibilidades sociales.

La dualización mente-cuerpo, naturaleza-cultura, es la base de toda una matriz epistemológica de la que se deriva, al menos en parte, la crisis civilizatoria actual. De esta división nace la idea de "progreso" como el éxito manifestado en superar las limitaciones del mundo natural, siendo este algo a conquistar. Y de esa misma idea, a pesar de que nacen muchos de los "avances" que resultan en no ser ya víctimas de las inclemencias de la vida a la intemperie, nace también la cadena de pensamiento que nos ha traído a la situación presente.

Basada, además, en una radical desigualdad, entonces el humano ha encontrado en la jerarquía social el modo de que algunos puedan desplegar un modo de vida opulento, mientras grandes poblaciones no solo viven las citadas inclemencias de "lo natural", sino que soportan los resultados de dichos niveles de consumo y su consecuente contaminación.

Entonces, con miras a enriquecer la comprensión de las sensibilidades sociales situadas, desde diversos abordajes podemos complejizar la noción de "ambiente", poniendo en tensión ciertas ideas dominantes, desde la modernidad, hacia/desde un pensamiento ambiental del "sur". En palabras de Machado-Aráoz, hablamos de una crisis ambiental - crisis del proyecto moderno - colonial en su dimensión política ("inviabilidad de patrones de consumo dominantes"), y epistémica ("crisis de un sistema de representación, crisis en la forma de conocer y percibir”): “[...] Remontándose a la tradición judeo-cristiana, la Ilustración radicalizó las separaciones entre lo sagrado, lo humano y la naturaleza, instituyendo diferentes regiones ontológicas (lo físico y lo metafísico) y estableciendo una correlativa ruptura epistemológica entre mente y cuerpo y entre filosofía, ciencia y religión (Lander, 2000)" (Machado-Aráoz, 2010, p. 36). ${ }^{6}$

Sin dudas, esta crisis es ampliamente reconocida por la mirada hegemónica y los grandes organismos que manejan la "agenda ambiental". No obstante lo cual, el modo como es expresada

\footnotetext{
${ }^{6}$ En dicho escrito, el autor realiza un recorrido por demás nutritivo sobre las nociones de naturaleza de la modernidad, donde la ciencia cumple un rol central en alcanzar su dominio. De allí que este camino, por momentos algo ecléctico para el lector, resulta importante para nuestras posibilidades de generación de conocimiento situado: "La complejidad que caracteriza al colonialismo moderno reside en la eficacia performativa de su episteme: la conquista militar, la subyugación política y la explotación económica de los pueblos (cuerpos y territorios) subalternizados, no ha sido sino efecto y condición de la conquista semiótica de la naturaleza, aún la de la naturaleza humana.” (Machado-Aráoz, 2010, p. 36).
} 
refuerza la dicotomización, y la consecuente salida productivista-racional propia del capital. Según hemos expresado en otro lado: "el discurso global en su concepción 'genérica' de la problemática, tanto en su diagnóstico como en sus líneas para la acción y la 'política', aún reconociendo en su retórica a otros saberes, culturas, etc., se impone sobre ellas en su cometido universal (del capital) imponiendo una forma (mercancía) en la apropiación y producción de la naturaleza" (D'hers et al., 2018, p. 8). ${ }^{7}$

Autores como Phillipe Descola (2002), entre otros, retoman cosmogonías propias de algunos pueblos del Amazonas para ejemplificar culturas que solo ven una diferencia de grado entre lo animal y lo humano. Pueblos "no-modernos" perciben, a su vez, animales que poseen alma, por lo que no se podría hablar de una división estricta:

Dentro de este cuestionamiento radical, el autor [Descola] propone reemplazar la dicotomía naturaleza-cultura, solo relevante en las lenguas europeas, por las relaciones de los humanos entre ellos y con los no humanos a partir de la distinción entre una materialidad y una interioridad. Según las combinaciones posibles entre estos dos elementos, sería posible distinguir cuatro grandes tipos de ontologías: el totemismo, el animismo, el analogismo y el naturalismo... La idea de naturalismo presupone, como afirma Descola, una discontinuidad de las interioridades entre animales y humanos, a la vez que una continuidad material. Desde Darwin en adelante, el naturalismo instaura una organización cosmológica [...] (Carman, 2007, p. 20). ${ }^{8}$
Es así que María Carman (2007), retomando estas discusiones, hablará de "gradación de humanidades" para poder captar el fenómeno de la violencia implicado no solo en un posible abuso de "lo natural", sino en lo que creemos es el punto central: violencia sobre otros humanos "no tan humanos".

Volviendo al hilo teórico referente a las sensibilidades en relación con su espacio vital, sin poder profundizar en esta discusión igualmente relevante, huelga decir que, concretamente, no buscamos tampoco hacer una oda - romántica - a un mundo previo e intocado, sino que trabajamos en comprender aquello que sostiene (teórico-epistemológicamente) esta configuración, a nivel de las sensibilidades y el espacio vivido. Antes que para un "retorno" a un estado previo, para desentrañar cómo subsistir en este siglo, en el que la palabra unión en sentido amplio - y unión como poner en relación/explicitar relaciones posibles - deviene clave, vital y epistemológicamente.

En este contexto, la misma noción de límite está puesta en cuestión. En primer lugar, la noción de límites y bordes entre el cuerpo propio, el ambiente, y sus mutuas configuraciones, según veremos más adelante. En segundo lugar, la definición de límite, así como se revisa la distinción naturaleza-cultura, se pone entre paréntesis la distinción entre "lo natural" y "lo artificial": la naturaleza como la imaginamos, ya no existe. En términos de Donna Haraway:

\footnotetext{
${ }^{7}$ En el citado trabajo se realiza una revisión de la literatura referente a "lo ambiental" en tanto problemática de la agenda política mundial, definida en términos de las Conferencias y Cumbres internacionales desde los años 1970. En dicho contexto, se estableció el "desarrollo sostenible" como salida y solución a la "problemática ambiental". Es así que en este artículo buscamos en cierto modo, retomar el sentido de lo ambiental en término amplio, en tanto relación "sociedad-naturaleza", superando la visión empresarial-estatal, en un intento de aportar hacia la reapropiación de las energías vitales. Esto dicho en dos sentidos: tanto en lo que vemos como "entorno", como en nuestro "propio" territorio -corporal.

${ }^{8}$ Tomamos la recepción de Descola en Carman, dado que es una autora local que viene analizando las vinculaciones naturaleza-cultura hace tiempo. Creemos pertinente observar la "recepción” de las concepciones que con respuestas a la mirada hegemónica pero nacidas en el "centro", en el propio ámbito teórico en el que nos movemos. Del mismo modo, revisamos lo que Machado-Aráoz sintetiza de D.Haraway.
} 
Haraway (1991) marca la transición de la etapa 'ecológica' del capital, a otra de naturaleza plena e internamente intervenida por el capital; una naturaleza que emerge como tecnológicamente producida, donde los límites entre lo orgánico y lo mecánico, lo 'natural' y lo 'artificial', lo 'biológico' y lo 'tecnológico' tienden a borrarse completamente (Machado-Aráoz, 2010, p. 43).

Es así que muchas de nuestras definiciones e incluso aspiraciones, ya no tienen asidero en lo real. Teniendo en cuenta la espacialidad, vivimos en un entorno completamente modificado, tanto en la ciudad como en el campo. Ambos términos son un continuo, funcional, que no puede ser comprendido el uno sin el otro. Lo que debemos analizar son sus relaciones, incluso el rol que tiene hoy el lugar natural por antonomasia como es la citada selva amazónica, dentro de los proyectos políticos a corto plazo. Entonces, antes que analizar los “entornos naturales" en sí, el siglo XXI exige generar escenarios a futuro a partir de la comprensión de estas configuraciones mutuas; y en el nivel estructural, desde el capital internacional en tanto es el que regula y modela los hábitats y "entornos" a nivel mundial. Asimismo, y cada vez con más fuerza, genera modelos de "gestión de la crisis ambiental", para mantener los cánones de explotación y ganancia. ${ }^{9}$
Retomando el punto de conexión entre espacio, hábitat y sensibilidad, podemos afirmar entonces que ya no hablamos de naturaleza en sí, como escindida de las configuraciones culturales, sino de un continuo, que es regulado por el capital, y dispone de los “entornos" según la geopolítica dicte. ${ }^{10}$ Luego, yendo más allá, pensamos el espacio vital profundamente configurado por las sensibilidades sociales. Para comprender esta relación, referiremos ahora a algunas líneas teóricas que vienen generando conexiones entre afectividades y espacialidades.

El primero que refirió a la relación mutua entre espacialidad y sentidos fue Yi-Fu Tuan (2007), quien puso el foco en la espacialidad de los sentidos y su rol en formar la relación afectiva de las personas con su hábitat. Señaló la distinción entre un espacio indiferenciado y un lugar, que deviene tal a medida que lo conocemos a través de nuestros sentidos.

Luego, sintéticamente, desde lo que se dio en llamar antropología de los sentidos, David Howes va más allá de la noción de embodiment (según lo delineado por Thomas Csordas, véase D'hers, 2017; D’Angelo, 2016), hacia la idea de emplacement, apoyándose en la interrelación sensual entre cuerpo-mente-ambiente, donde se tiene en cuenta al cuerpo-haciendo como parte de la "ecología de

\footnotetext{
9 Nuevamente, apoyándonos en la mirada clara de Machado-Aráoz: "Desde la conformación y expansión histórica del capitalismo como sistema mundial, la 'naturaleza' y los procesos ecológicos todos, así como los cuerpos y las agencialidades humanas, son resignificados y transformados en tanto objetos y medios de trabajo en el proceso de formación de valor. Desde entonces, 'es el proceso de acumulación y expansión del capital lo que condiciona el funcionamiento, la evolución y la estructuración de los ecosistemas, así como las formas técnicas de apropiación de la naturaleza [y de las corporalidades humanas]' (Leff, 1994: 139)." (Machado-Aráoz, 2010, p. 40).

${ }^{10}$ Abriría otra enorme discusión lo propuesto desde el ecofeminismo, en sus múltiples formas y propuestas (radical, feminismo ecologista, etcétera). A efectos de este escrito, solo referimos a la mirada de Carolyn Merchant, quien remarca la identificación naturaleza-femenino, por un lado por la maternidad como posibilidad, a la vez que salvaje y dominada por las pasiones, incluso amenazante, frente a la racionalidad masculina. De allí el dominio del hombre tanto sobre lo natural como sobre lo femenino. Dentro de la enorme discusión teórico-práxica que plantean, encontramos a Vandana Shiva, justamente en India, quien ve la explotación y destrucción de la naturaleza como intrínseca al modelo de desarrollo propio de la industria moderna en tanto imposición colonial, como ya dijimos. El par desarrollo-crecimiento económico (masculino) se impuso por sobre lo natural (en la cosmogonía india, la naturaleza como madre tierra-femenina), en tanto "pasiva" y a ser dominada. Véase Llort i Juncadella, 1994; D'hers et al., 2018.
} 
las cosas en progreso" (Howes, 2005, apud Pink, 2011, p. 344). ${ }^{11}$ Básicamente, postulan que esto permite verlo como un organismo, es decir, en relación. Esto es, habilita reconocer la especificidad e intensidad del "lugar" y sus contingencias, junto con su historicidad. ${ }^{12}$

Vemos cómo estas visiones se vinculan, finalmente, con el pensamiento complejo en tanto síntesis de lo que venimos exponiendo:

El paso de la perspectiva de la simplicidad propia del pensamiento moderno hacia el pensamiento complejo conlleva la necesidad de gestar nuevas cartografías, y sobretodo nuevas formas de cartografiar. En este contexto vital es preciso inventar otros instrumentos conceptuales y crear nuevas herramientas que nos permitan navegar territorios móviles y espacios multidimensionales. La metáfora de la red es una de las más fértiles para dar cuenta de la forma en que se nos presenta el mundo en el que estamos embebidos. A diferencia de la perspectiva moderna que exige un sujeto enfrentado al mundo y exterior a él, la mirada de las redes está inmersa en el mundo y se mueve a través (Najmanovich, 2007, p. 73; destacados nuestros).

En tanto dimensión de la actividad es que nuestra mirada sobre "el entorno", la naturaleza, necesariamente se transforma. Relacionalidad, intercambio, no-linealidad, todas caras de un caleidoscopio configurado siempre de manera dinámica por nuestros sentidos de la percepción y los significados que a ello atribuimos/encarnamos.

Tal como lo sintetiza Denise Najmanovich, autora argentina doctorada en Brasil, desde este abordaje han trabajado múltiples teóricos como
Francisco Varela y Humberto Maturana (2003), quienes indagan el carácter poiético de cada acción y experiencia. Según desplegaremos, la idea básica (y que encuentra vinculaciones entre neurociencia y budismo, algo que Varela particularmente profundizó) es que toda cognición no es representación, sino ir activamente creando ese mundo a medida que se acciona.

Es a partir de esta síntesis que abordaremos ahora brevemente la noción de cuerpo/emoción, en sintonía con lo expuesto respecto de falsas aporías (naturaleza-cultura), para luego poder avanzar sobre el punto central del trabajo, la posibilidad de una práctica/entrenamiento y disciplina corporal-afectiva en tanto integración posible hacia - valga aquí la expresión de deseo - una reapropiación de energía vital.

\section{Sensibilidades otras}

\subsection{Cuerpo como terreno a dominar vs. cuerpo/emoción como territorio y totalidad}

El recorrido anterior, aunque breve, nos permite dar paso a la articulación entre la mirada sobre la naturaleza, y el cuerpo en tanto parte de ella. Es en el cuerpo donde se plasman, vitalmente, estas dicotomías que marcan caminos posibles de experienciación.

Desde la tradición judeo-cristiana, según ya dijéramos, el mundo sensible queda escindido de la mente, de jerarquía menor y atado a las pasiones,

\footnotetext{
${ }^{11}$ En este sentido, llegando a las últimas líneas de investigación en vinculación con lo ambiental, podemos referir no ya sólo al embodiment como asumido dentro de la literatura más especializada, sino al concepto "toxic embodiment", desde el "nuevo materialismo" que aspira a estudiar los efectos, aún insospechados, de la exposición permanente a nuevas hormonas en los alimentos, ciertas condiciones de consumo y de habitabilidad particulares, entre otros fenómenos. Véase http://newmaterialism.eu/updates/cfp-toxic-embodiment

${ }^{12}$ Según propusimos la pregunta en otro lado, la percepción en tanto objeto ha sido tratada desde múltiples perspectivas (D’hers, 2017). Solo detallamos algunas, dado que el objetivo es otro.
} 
víctima de un equívoco fundamental. Es así que, al día de hoy, hemos llegado a una vuelta al cuerpo dentro del mundo urbano, con cierta explosión de la atención y el "foco" en el entrenamiento, pero atravesada por el paradigma de dominio y eficiencia, propio de la modernidad. Siguiendo con la línea expuesta en el apartado anterior, podemos pensar que el dominio técnico-corporal se corresponde con el paradigma moderno de dominio sobre la naturaleza (interna y externa, "naturaleza capitalizada"), en términos de Machado-Aráoz (2010, p. 44). ${ }^{13}$

Dicho esto, a nivel del estudio del "cuerpo", entendemos que ya en las ciencias sociales hay un consenso en torno a la relevancia de los estudios de los cuerpos y sensibilidades sociales. En este campo, hemos trabajado apoyándonos en una sistematización teórica que sintetiza la mirada desde lo que se denomina "cuerpo/emoción" en tanto objeto de estudio de la sociología (Scribano, 2013). ${ }^{14}$ Teniendo en cuenta que "el ser humano se auto-percibe, es percibido y se auto-representa como 'inmediatamente' en un cuerpo. Pero también una guía para rastrear los modos de dominación que parten y llegan a esos cuerpos 'explicados' como pura naturaleza” (Scribano, 2013, p. 102, destacados nuestros) es que volvemos sobre este proceso de percepción como actividad situada, en tanto momento cognitivo-afectivo central a la hora de explicar lo social "hecho cuerpo(s)".

Dentro de este campo, y tomando como punto de partida lo expuesto en otro lugar, podemos afirmar sintéticamente que, en línea con la fenomenología de Maurice Merleau-Ponty definimos cuerpo propio como una construcción intersubjetiva, dinámica, donde operan una conciencia constituyente y conciencia perceptiva (véase D'hers, 2017, p. 144). ${ }^{16}$ Aquí, la percepción no es una sumatoria de eventos que se adicionan a la experiencia, sino una operación témporo-espacial permanente, una construcción de sentidos no lineal, formada por procesos en constante interacción e intercambio con el "entorno". Entorno ya atravesado sensorialmente, inescindible de la experiencia que es configurada a la par que lo modifica.

Es decir, entorno como ambiente, resultado y antecedente de la relación entre ese "mundo natural" y el "mundo social", solo divisibles analíticamente. Sujeto como totalidad, enactiva:

\begin{abstract}
El sujeto no es ya una entelequia racional, sino una dimensión de la actividad de una persona de carne y hueso, viva y activa, en permanente transformación a través de los intercambios con su ambiente con el que está embebida y con el que co-evoluciona de forma no-lineal (Najmanovich, 2007, p. 73; destacados nuestros).
\end{abstract}

\footnotetext{
13 “como la crítica marxista permite alumbrar, es el propio Capital, impostado en el lugar del Sujeto, el verdadero conquistador/colonizador del mundo. La organización colonial del mundo no es sino la configuración del capitalismo como nuevo régimen eco-bio-político de producción del mundo; es el propio capital el que instituye las formas modernas de representación, apropiación y disposición tanto de la naturaleza interna (cuerpos, trabajo, agencialidades) como de la naturaleza externa (materiales, energía, territorios) para la realización de la acumulación del valor." (Machado-Aráoz, 2010, p. 40).

14 Tanto para este binomio, como para lo desplegado previamente de naturaleza y cultura, a pesar de cierta diferencia de carácter en ambos pares, nos hacemos eco de las palabras de Scribano $(2013$, p. 8) al advertir "No es pretensión del presente escrito negar la existencia de los subcampos disciplinares, ni tampoco hacer una llamada a su eliminación; nuestra argumentación se concentra en la presentación de una separación imposible y de sus consecuencias teóricas y políticas".

15 Profundizando ese planteo, hemos expresado que "Desde el campo de las discusiones actuales dentro de la fenomenología, remitimos a autores como Shaun Gallagher, quien trata el tema de cómo la conciencia (awareness of the body) influencia a la experiencia. Vittorio Gallese referirá a la importancia de la empatía. Vinculados también con Husserl y la fenomenología, insisten en el rol del cuerpo actuando en la acción de percibir ('there can be no perception without awareness of the acting body' Gallese, 2001:43). Así es como "la conciencia de nuestro cuerpo en acción no puede estar escindida de los mecanismos que organizan/controlan la acción” (D’hers, 2018a, p. 79).
} 
En efecto, según afirman Colombetto et al. (2018), cada vez más dentro de las ciencias cognitivas se pone en cuestión la idea de que podríamos comprender cómo funciona la mente solo observando el cerebro. ${ }^{16}$ Refieren a autores como $\mathrm{S}$. Gallagher (véase D'hers, 2018a), Thompson (que referimos más abajo), Shapiro, entre otros, donde se afirma que las capacidades psicológicas están atadas al cuerpo, y al modo en el que interactúan con el ambiente material y social. Llamados abordajes "4E", entienden que las capacidades están embodied (encorporeizadas, ligado a la psicología y la agencia), embedded ("incorporadas, incrustado, arraigado", situadas dentro de un ambiente que las limita y contiene), enacted (literalmente, establecidas, definidas como reflexivas, reflejan la actividad de dar sentido y crear sentido de las personas), y quizás incluso extended, es decir, "extendidas" más allá de la cabeza por los recursos materiales y sociales.

En esta línea, Glowinski et al. (2017) realiza un aporte a los estudios de la percepción al revisar las vinculaciones entre cuerpo, espacio y emociones, aunque lo delimita al análisis de la kinesfera y las posibilidades de lectura de las emociones vinculadas a diversos movimientos por un observador. ${ }^{17}$

Excedería estas páginas revisar la literatura referente a la temática, pero sí podemos afirmar que debemos indagar en torno a cómo la mente actúa como un sentido de percepción y no como algo escindido de la experiencia, que solo sintetiza y organiza la información percibida. Este "proceso perceptivo, tendrá directa incidencia en las posibi- lidades de percibir o no, algo, sea eso "interno" o "externo", del entorno. Para poder reflexionar desde/sobre este "devenir entramado", Najmanovich insiste en apoyarse en la empatía, una "curiosidad abierta, sin fronteras, afectiva, emotiva", frente a la deslegitimación de toda percepción que no entre en las definiciones modernas:

los enfoques complejos caracterizados por pensar en términos de interacciones no lineales nos dan la posibilidad de salir del círculo vicioso y habilitar un pensamiento fluido (...) El conocer, entendido como configuración que surge de la interacción multidimensional, ya no es un producto rígido y externo cristalizado en una teoría, sino una actividad. La configuración surge del encuentro de los seres humanos con el mundo al que pertenecen, encuentro múltiple y mediado, en él emergen simultáneamente agente productor del conocimiento y el mundo en su mutuo conocerse y hacerse, en un devenir sin término (Najmanovich, 2007, p. 74).

Según ya hemos explicitado, esta mirada retoma lo propuesto por Varela y Maturana, que remarcan que las redes se tratan de dinámicas no lineales, procesos antes que estructuras fijas o productos. Es así que lo único que distingue a cualquier "sistema organizado" del entorno es que produce una forma específica de delimitarse a sí mismo, generando cierta autonomía relativa; sin formas establecidas a priori (Najmanovich, 2007, p. 80; Varela \& Maturana, 2003).

Tal como ya hemos referido en otro escrito, desde la neurofenomenología, estos autores postulan que el cuerpo se define como "enactivo" -todo

\footnotetext{
16 “'Affectivity Beyond the Skin' is an investigation of some of the many ways affectivity can be scaffolded by resources beyond the head. Each contribution challenges the common view in emotion science that affective states can be characterized by focusing exclusively on internal states of an individual's brain and body." Así sintetizan la propuesta editorial.

17 "alternative approaches have been developed more in recent decades. Research results in psychology suggest, in particular, that body movements do constitute a significant source of affective information (Wallbott, 1998).” (Glowinski et al., 2017, p. 34).
} 
hacer es conocer y todo conocer es hacer (D'hers, 2017, p. 147). Esto se encuentra en estrecha relación con la concepción budista de la conciencia, postulando que la mente no puede ser "separada" del cuerpo, sino que todo son procesos corporizados. A la vez, la noción de unidad es eje de este modo de trabajar sobre la experiencia en sentido amplio.

Profundizando en la crítica a la modernidad, siguiendo lo que introdujimos más arriba, debemos cuestionar la noción de límite:

La cuestión entonces, se organiza en términos de cómo abordar el análisis de ese "perceptor encarnado" para poder acercarnos a su mundo vital particular, a su modo de construcción del entorno: "más acá" de la materialidad de dicho entorno, desdibujando la idea de límite y abriendo la complejidad de los bordes (Bhaskar, en Scribano, 2011), que se constituyen individualmente - a través de experiencias - sociales -, interesa el modo cómo ese sujeto lo experiencia, entendiendo ese momento como una construcción intersubjetiva, y realizando esa construcción a través de un refinamiento de la experiencia de sí desde la observación conjunta, en el marco de las unidades de experienciación” (D’hers, 2018a, p. 82).

Vemos una vinculación en esta apertura hacia los bordes (en línea con lo propuesto por Scribano, 2011) como un peldaño más en la crítica de la modernidad: "Para el modelo de pensamiento que hemos heredado el concepto de límite se establece según oposiciones insalvables entre términos completamente puros en sí mismos y a la vez radicalmente independientes: lo propio y lo ajeno, el Yo y el Otro, adentro y afuera" (Najmanovich, 2007, p. 79). ${ }^{18}$ Es así que ya no rastreamos eso que está "dentro" o "fuera", definir claramente lo real en tanto se corresponda e identifique de una vez y para siempre con lo que nuestras categorías han hecho de ello. Desde los límites nos acercamos a los bordes, para poder hacer visible/perceptible en sentido amplio, nuevas texturas de lo real.

Dentro de este constructivismo, vemos como el conocimiento no es algo que exista a priori (a modo de categorías del sujeto) ni en tanto parte del objeto (empirismo), sino en la relación, según venimos desplegando: "el conocimiento surge en un proceso de organización de las interacciones entre el sujeto ('sujeto de conocimiento') y esa parte de la realidad constituida por los objetos ('objetos de conocimiento') (García, 2000, p. 61)” (Acevedo, 2011, p. 281).

¿Cómo es ese proceso en nuestra relación cuerpo-mente? ¿Cómo acceder a su estudio? Cómo varía atravesado por múltiples prácticas de entrenamiento y "autoconocimiento"?

Si bien estas preguntas son imposibles de responder en este estadío de la investigación, pasemos ahora a delinear algunas vinculaciones con la práctica vista como parte de ciertos modos de "entrenamiento", pero centrada en el autoconocimiento como es el yoga, según iniciamos ya este camino de indagación. Nos hemos preguntado por las posibles conexiones entre percepción, afectividad (D'hers, 2017, p. 139, nota al pie 7), luego desarrollando posibles vinculaciones desde la propia experiencia (D'hers, 2018a).

Continuando con el hilo desplegado, llegamos aquí a la cuestión de cómo podemos relacionar estas miradas de lo ambiental, desde la complejidad y una crítica al pensamiento moderno colonial, hacia la noción de conciencia desde un paradigma

\footnotetext{
18 "Desde una mirada dicotómica, el límite es siempre fijo y separa drásticamente un exterior y un interior ("lógica conjuntista identitaria, como Castoriadis los bautizó). Los principios de identidad, no contradicción y tercero excluido, forjaron un modo de definición que establece límites infranqueables y elementos aislados." (Najmanovich, 2007, p. 79).
} 
diverso, para ampliar nuestro entendimiento sobre la percepción. Todo esto aspira a comprender qué implica la noción de unión, y cómo podemos nutrir lo dicho hasta aquí desde otro abordaje y matriz epistemológica.

\section{2. ¿Integración cuerpo-mente-espíritu?}

Para enriquecer las posibilidades de comprender la "operación perceptual", si bien sabemos que hay ya variados enfoques, tanto desde estudios del cuerpo, de las emociones, de la conciencia, de la percepción en tanto actividad de los órganos sensoriales, así como multiplicidad de experimentaciones y caminos de indagación, optamos por continuar con el recorrido de conectar y poner en relación, y arribamos aquí a la noción de conciencia en sí. En continuación con lo expuesto anteriormente afirmamos que:

En esta dirección, podemos decir que el sentido de la 'mente' no trasciende a los sentidos corporales pero en cambio, los complementa, los compone. El sentido de la mente "siente" objetos mentales, tanto como el sentido del tacto puede tocar y percibir a través de la piel, la presión, etc. No hay ya división entre un plano mental e imaginario y otro real y físico. Más bien, todo dentro del realismo, es ilusorio, por lo que toda experiencia emerge del contacto entre los sentidos (incluida la mente) y los objetos (implicados también los propios objetos mentales, los propios horizontes de sensibilidad imaginados por el sujeto). Entonces, debemos volver crítico este proceso de configuración (sensible, mental, emocional) de la percepción, histórica y social, antes que personal y "propia” (D'hers, 2018a, p. 80-81).

Brevemente, como un paso más en este "volver crítico" este proceso, abordamos la disciplina y filosofía del yoga en tanto camino hermenéutico y

de apertura de comprensiones otras sobre lo que venimos refiriendo como elementos "modernos" como son naturaleza, cuerpo, cultura, en tanto compartimentos estancos y fijos. ${ }^{19}$

Sin perder categorías, antes bien vinculándolas, es que culminamos este recorrido que partió de una certeza de no-adecuación de las categorías modernas del mundo respecto de la experiencia que queremos comprender, para navegar por algunas relaciones posibles en torno a la percepción y el ambiente, y arribar a ciertas reflexiones que enraizan en experiencias vitales que nutren la comprensión del mundo-como-se-presenta: la vivencia de un desdibujamiento de los propios límites, las conexiones permeables entre el cuerpo propio y ese "entorno", del que tenemos ciertas representaciones, y a la vez somos-ahí.

Cómo dinamizar este vínculo cuerpo-entorno, en tanto todo es conformado/conforma el ambiente en el que la vida se desenvuelve, no linealmente, interconectado, en esto que ya-es-ahí y a la vez, es-en-proceso, en operación y no como imputaciones de sentido definitivas de un sujeto hacia un objeto. Cómo funciona, en última instancia esto que hemos definido como límite (naturaleza, cultura, cuerpo, mente), al volverlo poroso en nuestra propia "mente", en nuestro entendimiento de lo real.

En primer término, el yoga en tanto práctica se encuentra en expansión (D’hers, 2018b). Si bien no se puede argumentar que es "masivo", sí creemos, según ya hemos expuesto, que reviste interés por su aporte en tanto modo particular de definición y entendimiento del cuerpo/emoción. Según se describe en los Yoga Sutras de Patañjali, el yoga se define, en Sánscrito, como citta vritti nirodaha, suspensión o reducción de las fluctuaciones/mo-

${ }^{19}$ Hemos revisado preliminarmente la temática ligada a los sentidos de la percepción, cuántos son, cómo operan, en D’hers (2018a). 
vimientos de la conciencia, citta, compuesta por manas - mente, buddhi - inteligencia, y ahamkara - ego. En principio, la conciencia es así definida como vehículo de la observación, atención, intención y razón. Tiene tres funciones: cognición, volición y movimiento (motion) (D'hers, 2018a, p. 77). Este es el camino (sadhana, práctica) hacia la conciencia pura (Samadhi) y más allá de la división entre lo visto y el que vé (división S-O, y el sujeto en tanto objeto para sí mismo, objeto de representaciones, sensaciones, etcétera). Esto es solo posible a nivel de la experiencia, dado que cualquier explicación sería volverlo objeto reflexivo.

Respecto de estudios sobre la práctica del yoga en particular, primero cabe destacar el nuevo rol que India (cuna de dicha práctica) en tanto nación cumple para la geopolítica mundial y la provisión de recursos al capital internacional. Dentro de este mapa, autores como McDonald (2003) rescatan las intenciones del nacionalismo indio en cuanto a valorizar ciertas prácticas ("embodied practices") como signo definitorio de una identidad, a la vez milenaria y central en la configuración global actual. El autor analiza prácticas ligadas a entrenamiento (shakha y kalarippayattu, arte marcial del sur), que aspiran a la realización del self a través de arduas prácticas hacia un estado de conciencia de cuerpo-mente donde el cuerpo se convierte en todos los ojos. A través de dicho análisis vincula la posición actual del país en el marco de la globalización occidental, y el surgimiento de un nacionalismo agudo. De allí que creemos, en conexión con el primer apartado, que es clave comprender la geopolítica desde/atravesada por las sensibilidades, y viceversa.

Luego, es claro que hay multiplicidad de estudios acerca de la práctica del yoga en general, y de las evidencias de sus efectos en la conciencia en particular. Por ejemplo, a nivel local hemos encontrado un texto con el que este escrito resuena. ${ }^{20}$ Dentro del campo de los estudios de la disciplina, D’Angelo (2016, p. 22) propone "analizar el sentido que las categorías 'cuerpo', 'mente' y 'espíritu' guardan en el marco del Yoga y los vínculos que los practicantes más avezados establecen entre ellas bajo la categoría 'conciencia' resulta necesario un breve recorrido conceptual". Conceptualmente en línea con lo que venimos proponiendo, la autora parte de una revisión teórica pertinente de la dualidad cartesiana mencionada más arriba, observando que "su error no habría sido la diferenciación entre sustancia pensante - res cogitans - y sustancia física - res extensa -, sino el establecimiento de límites definitivos entre los procesos intervinientes o la imposibilidad de ver 'el fantasma en la máquina' (Ryle, 1967)" (D’Angelo, 2016, p. 23).

Es importante referir a la diferencia que la autora marca en cuanto a dualismos en 'Occidente' y 'Oriente'; dado que según algunos intérpretes de los textos clásicos como los Yoga Sutras, esta es una cosmovisión dualista pero en sentido de dividir conciencia o espíritu (pūrusa) de materia (prakriti), donde están incluidos lo que conocemos por cuerpo, mente, respiración (D'Angelo, 2016, p. 26). Un punto clave de este trabajo es el modo como busca no caer nuevamente en el dualismo, al referir a unión cuerpo-mente, dado que la filosofía no lo plantea así (más allá del modo como sí lo expresan quienes lo practican).

Pero sí debemos reconocer que sería más bien una unión en términos de cambio cualitativo: a través de la práctica del yoga y meditación, el ser integra el citta (ya referida 'conciencia': mente-in-

${ }^{20}$ Para ver otras referencias, no exactamente ligadas a la temática pero sí a la disciplina aquí tratada, véase D’hers (2018b). 
teligencia-ego) con el cosmos. Entonces la clave está en interpretar dentro del citta al cuerpo en tanto compone esa "inteligencia", en tanto cambio de estatuto cosmogónico. Al pausar las fluctuaciones del citta, ya en acto nos unimos con eso que es el universo, más no sea por una exhalación. Pero es una ardua tarea salir del propio dualismo en cada lectura de los textos, tarea que aspiramos a reforzar con este escrito.

Evan Thompson (2015) trata el tema desde el punto de vista de las influencias entre la filosofía india, sus múltiples escuelas de pensamiento, y la neurociencia moderna, advirtiendo que se pueden nutrir mutuamente para aportar al entendimiento de cómo funciona la conciencia en general, y los momentos en que el "yo" está presente o ausente, en particular - refiriendo por ejemplo, a los estados de sueño y las diversas etapas que lo definen.

Smith (2007, p. 41), por su parte, revisa justamente la relación entre cuerpo, mente y espíritu, en la práctica del yoga, concluyendo que la práctica en sí es una herramienta fenomenológica para la experiencia de aspectos del "embodiment" que han permanecido en el plano conceptual, reforzando lo que aquí se plantea: "La conjunción de la práctica del yoga y una fenomenología del cuerpo infligida culturalmente, puede proveer de otro 'encuentro' que resulta en el enriquecimiento de ambas formas de investigación sobre el carácter encorporeizado de nuestra existencia en-cuerpo". ${ }^{21}$

R. Rao (2005) marcará la distancia entre la conciencia y la mente, como fundamentalmente diferentes. La realidad está compuesta, desde los sistemas Vedanta del que el yoga es parte, de ser (being - sat en Sánscrito), saber (knowing - cit) y sentir (feeling - ananda). En esta línea, según este autor, la conciencia se vincula con el conocimiento, no material, mientras que la mente es física. Es así que, conectado con lo postulado más arriba, la conciencia no interactúa con la mente, el cerebro o cualquier otro proceso físico. La mente es la interfase que conecta la conciencia con el cerebro y el resto del mundo físico. Es así que Rao \& Rani (1994) han analizado el aumento de la conciencia corporal (body awareness) en un grupo de practicantes de yoga.

Particularmente, ahora, nos acercamos al trabajo de J. Morley (2001, p. 74), quien analiza las vinculaciones entre la respiración, la práctica del yoga, y la fenomenología del mencionado Merleau-Ponty, en tanto aquí se sintetiza lo que venimos delineando, en términos seguramente más cercanos al lector que la propia práctica. Morley vincula así la epoché (o reducción fenomenológica, suspensión de toda creencia o duda), típica del abordaje trascendental husserliano temprano, como "convergente" con las prácticas de meditación.

Y aquí el autor ve una relación directa con el modo como Husserl diferencia a un yo ("I") de la subjetividad trascendental de un ego inmerso en la división sujeto-objeto. Asi es que desde ambas perspectivas, se busca una transformación de las "estructuras mentales" de la percepción hacia el desarrollo de una conciencia testigo reflexiva (reflexive witness consciousness) de nuestro propio proceso de percepción del mundo. Y el problema que encuentra en ambas, es un "residuo" de un ideal puro de conciencia, un a priori o pura subjetividad distinta de un mundo externo, objetivo. El autor llega así a revisar los postulados de Heidegger, quien marca que es la relación entre el sujeto y el mundo la que es previa a la división categorial: "Los seres humanos

\footnotetext{
21 “The conjunction of the practice of yoga and a culturally inflected phenomenology of the body can provide for another 'encounter' that results in the enrichment of both forms of inquiry into the character or nature of our embodied existence" (Smith, 2007, p. 41).
} 
están inmersos dentro y emergen de la existencia de manera general. El concepto de Heidegger de dasein (literalmente, "estando-ahí"), traducido típicamente como ser-en-el-mundo, es ese de la existencia situada históricamente que es conciente de sí existiendo en un horizonte temporal finito, esto es, hacia la muerte" (Morley, 2001, p. 74). ${ }^{22,23}$

Arribamos nuevamente a la noción de cuerpo vivido, desde la mirada del mencionado Merleau-Ponty, quien "resalta la experiencia sensorial humana como emblema de los antónimos metafísicos tales como sujeto y objeto o interioridad y exterioridad. Su término sentido [sens] connota ambos la experiencia sensible y el sentido [meaning-en tanto dar significado-]" (Morley, 2001, p. 75). ${ }^{24}$

Cuerpo vivido, "flesh" (carne), conciencia encorporeizada (y no ya delimitada a una "identidad"), nexo entre los roles de ser agente activo de la percepción, y objeto pasivo de la percepción por parte de otros. La "carne" implica esta relación, no se vive como S-O, ni interno ni externo, pero ambos en proceso constante de transformación. Es aquí que el autor ve el vínculo, una vez más, con la práctica yóguica, apoyada en la relación entre el yo y el mundo como medio de meditación: "El control del cuerpo está igualado/asimilado con la maestría sobre la naturaleza externa, y este control es alcanzado enfocándose en los sentidos" (Morley, 2001, p. 75). ${ }^{25}$

En este proceso, nuestra percepción se ve "alienada" de la masa sintiente de nuestros cuerpos, y el pranayama, dirá el autor, la práctica de control de la respiración, va contra esta división, siendo la experiencia concreta del cuerpo, siendo-ahí, tanto interior y como exterior (Morley, 2001, p. 76). ${ }^{26}$ Aquí, la "propiocepción", la percepción en términos de conciencia vital de capas del cuerpo, que llamaríamos interno (texturas internas, diferencias entre partes blandas y duras dentro del propio "organismo", etcétera), se ve drásticamente agudizada. ${ }^{27}$

Finalmente, es importante referir a cómo esta mirada, según ya hemos adelantado, implica una revisión de la noción de espacio, llegando a que "El espacio delimitado del cuerpo es, casi en términos

\footnotetext{
22 "Individual human beings are immersed within and arise out of existence generally. Heidegger's concept of dasein (literally "there-being"), typically translated as "being-in-the-world," is that of a historically situated existence that is aware of itself as existing in a finite temporal horizon, that is, toward death." (Morley 2001, p. 74). Todas las traducciones son propias, por lo que se incluyen las versiones originales para que el lector pueda contar con el idioma del texto.

${ }^{23}$ Más aún, "Hence, in existential phenomenology, human existence is adhered to the world, neither an internality nor an externality, neither subject nor object, but a spatiotemporal openness. Merleau-Ponty's contribution was to tighten the concept of dasein through an existential-phenomenological rehabilitation of the human body." (Morley 2001, p. 74).

24 "Merleau-Ponty highlights human sensory experience as emblematic of metaphysical antonyms such as subject and object or interiority and exteriority. His term sens connotes both 'sense experience' and 'meaning')" (Morley, 2001, p. 75). Véase también Morley, 2010.

25 "Control of the body is equated with the mastery of external nature, and this control is achieved through focusing the senses" (Morley, 2001, p. 75).

${ }^{26}$ Concretamente: “'Outside' and 'inside' seem to imply the exactly rigid demarcation between the thing and the surrounding world that Merleau-Ponty is arguing against. At the same time, the terms 'inside' and 'outside' become semantically necessary when we engage with the body's spatiality." (Morley, 2001, p. 76).

27 "Proprioception constitutes one of these somatic sensations within the haptic system. In addition to proprioception, these somatic sensations comprise kinesthesia (the sense of movement) and the vestibular system (the sense of balance)" (Paterson 2009, p. 768-770). Tomando esta hecho de propiocepción en tanto ligada con el sistema háptico, confirmamos como la piel (ese límite que tratamos de visualizar desde otro punto de vista), y el tacto son centrales a la percepción y nuestra reflexividad sobre ella.
} 
yóguicos, una homología del cuerpo con el microcosmos y el mundo" (Paterson 2009, p. 768-770) ${ }^{28}$, reforzando la idea de que es esta diferenciación, solo a nivel de borde podríamos agregar, permite una empatía con los objetos, con el mundo "externo".

Es así que la corporeidad (corporeity), no es ya materia en sentido newtoniano, sino que se acerca al "elemento" (aire, tierra, fuego, agua, éter - que el autor no menciona). Así es como el cuerpo en este sentido, permite que ese "exterior" sea comprendido como propio. ${ }^{29}$ La cuestión central, entonces, es cómo esto opera, o se vuelve evidente, en la práctica, y si desde allí puede volverse "herramienta" dentro de nuestro campo de investigación.

\section{Reflexiones finales}

A modo de cierre del escrito, apertura de tantas preguntas, recordamos aquí que nuestra búsqueda se orienta a aportar a un saber (ambiental) emancipador, arraigado en los potenciales ecológicos y en la creatividad cultural de los territorios del sur (como propusimos en palabras de Leff, en la introducción). Partiendo de que nuestros territorios son parte de un saqueo y extracción de energía permanentes, el cuerpo/emoción como parte componente de ellos, es que trabajamos hacia un ensanchamiento de la comprensión de eso que nombramos y categorizamos como entorno, espacio, cuerpos, conciencia, mente..., viendo en esta filosofía y sus posibilidades cognitivo/ afectivas, una mirada otra para resignificar nuestro lugar como seres totales, en nuestro mundo vital.

Es así que, revisando las relaciones con lo material, y desde allí con lo interno/externo, la búsqueda de "unión" se convierte en comprensión de lo que ya está unido, ya es unidad. Esa unión (cuerpo-mente-espíritu en términos del yoga, unión con el entorno como seres vivos) debe comprenderse en el sentido de que ya está funcionando.

Si la filosofía hindú observa que es ya una unidad (prakriti), y lo que aspira es a reunir a esta materialidad con lo espiritual desde la práctica, podemos tomarlo como "herramienta", en principio hermenéutica, al menos. Es decir, un modo de definir y comprender que eso que veíamos como límites definidos (mente-cuerpo; naturaleza-cultura), recorridos en tanto parte de una unidad, con bordes dinámicos, nos permite ilustrar la unión con un "entorno" en crisis.

[Más aún, desde la misma práctica reforzar inclusive esa relación purusa-prakriti, y no ya solo la cultura del consumo y rendimiento.]

Es así que hablamos de unión en sentido amplio: poner en relación/explicitar relaciones posibles. A partir de conceptos como emplacement, atravesado por la mirada constructivista compleja, y de la perspectiva fenomenológica, encontramos resonancias con lo que la práctica del yoga propone, en el sentido más literal, hacia una vivencia de nuestra inmersión en el mundo.

Estas preguntas, a modo de aperturas, dejan ahora el terreno fértil para reformular ciertas aproximaciones al trabajo en terreno. Insistimos en que la propuesta aquí no fue el estudio de quienes practican yoga hoy, sino de qué preguntas otras podemos plantearnos como investigadores, a la hora de analizar cuerpo/emoción/espacialidad/territorialidad.

Dada la crisis ecológica y ambiental que atravesamos, y que se agudizará cada vez más, este

\footnotetext{
28 "The enclosed space of the body is, almost in yogic terms, a homology with the microcosm of the world" (Morley, 2001, p. 76).

${ }^{29}$ Finalmente: "In any case, once a body-world relationship is recognized, there is a ramification of my body and a ramification of my world and a correspondence between its inside and my outside, between my inside and its outside.” (Morley, 2001, p. 77).
} 
cambio de entendimiento puede devenir una pieza importante, en concordancia con muchos otros paradigmas y cosmovisiones, hacia la reapropiación de la energía vital en el marco de la depredación. Nos guía la pregunta por cómo dinamizar este vínculo "cuerpo-entorno", en tanto todo es conformado/conforma el ambiente en el que la vida se desenvuelve, no linealmente, interconectado. Todo "ya-es-ahí" y a la vez, es-en-proceso, en operación y no como imputaciones de sentido definitivas de un sujeto hacia un objeto. Entonces, más acá de que el poder financiero global es el que primero dispone de nuestras propias energías, sea desde miradas otras que aportemos a agrietar esta explicación única de la realidad y sus diagnósticos.

Y sea a partir de esta breve revisión epistemológica concerniente al ambiente y la naturaleza, y sus vinculaciones con la mirada sobre el cuerpo/ emoción, que nos preguntemos en última instancia acerca de la idea de "eficiencia" y dominio. Entonces, a través de una revisión del plano de conciencia y ambiente, cuestionando nociones como límite y entidad, y cómo afectan a dichas búsquedas de "dominio", ¿Qué es el dominio de sí, en el siglo XXI? O más bien, a qué nos enfrenta un mundo en crisis aguda, donde de la sensibilización depende la supervivencia. En esta línea es que, siendo el presente un estudio teórico preliminar en el que se sintetizan varias líneas de indagación, se abre aquí un desafío próximo de hilvanar estas preguntas hacia su posible aplicación en el trabajo en terreno.

Huelga decir que, en términos científicos, en este mapa recorrido como trama propuesta para hilvanar experiencias y revisitar cuestiones centrales a la hora de comprender las sensibilidades sociales en contexto, nos acercamos a la idea del paradigma constructivista ya citado, y presente en todos los campos científicos: la validez se apoya en el acuerdo y asunción de que toda interpretación-explicación es una atribución conceptual hacia/sobre la realidad, donde hay una interacción permanente entre eso que llamamos "teoría" y el mundo-ahí (Acevedo, 2011, p. 286).

Finalmente, aspiramos a que estos cruces colaboren a idear formas otras de generación de conocimiento, animando a reconfigurar caminos explicativos en un "mundo en crisis", cuando la palabra crisis ya pareciera no conmovernos.

\section{Agradecimientos}

Agradezco a Eduardo Galak por contactarme con Renato Izidoro, quien me dio esta posibilidad. Agradezco a Adrián Scribano, por sus aportes centrales tanto a este trabajo teórico, como al darme ánimos permanentemente en este camino de integración entre las huellas de mi experiencia y la práctica de investigación en ciencias sociales.

\section{Referencias}

Acevedo, C. Acuerdos comunes de validez: Diálogo entre la metodología cuantitativa y cualitativa. Cinta de Moebio 42, 276-287, 2011.
Carman, M. Las fronteras de lo natural y las fronteras de lo humano. Premio producción científica sobre la discriminación. Buenos Aires: INADI. 2007. 
Cerrillo Vidal, J. A. Medición de la conciencia ambiental: Una revisión crítica de la obra de Riley E. Dunlap. Athenea Digital, 17, 33-52, 2010.

Colombetto, G.; Krueger, J.; Roberts, T. Affectivity Beyond the Skin. Frontiers in Psychology, 9, 1307, 2018. Disponible en: https://www.frontiersin.org/articles/10.3389/ fpsyg.2018.01307/full

D'Angelo, A. Conciencia en la postura: del dualismo a la reflexividad en la práctica de Yoga. Revista Runa, 37(1), 21-38, 2016.

Daros, W. Religión y ciencia en el pensamiento de Albert Einstein. Revista INVENIO, 5(8), 45-56, 2002.

Descola, P. L'anthropologie de la nature. Annales. Histoire, Sciences Sociales, 1(57), 9-25, 2002.

D'hers, V. Tramando la percepción y la conciencia desde una experiencia del/con/hacia el cuerpo. Revista Controversias y Concurrencias latinoamericanas, 10(17), 73-89, $2018 \mathrm{a}$.

D’hers, V. ¿Intersticio o reproducción? Reflexiones en torno al yoga, el disfrute y la reciprocidad. Boletín Onteaikén, 25, 25-36, 2018b.

D'hers, V. Sentir (o del ser, saber, hacer). Reflexiones sobre la percepción. In: Scribano, A; Aranguren, M. (Orgs.). Aportes a una sociología de los cuerpos y las emociones desde el Sur. Buenos Aires: ESE Editora, p, 135-156. Disponible en: http://estudiosociologicos.org/-descargas/eseditora/ aportes-a-una-sociologia-de-los-cuerpos/aporte-a-una-sociologia-de-los-cuerpos.pdf, 2017

D'hers, V.; Veiguela, N.; Lovisa, C. Ambiente y desarrollo: ¿Un futuro sostenible? In: Actas de las IX Jornadas de Investigación en Antropología Social, UBA. Buenos Aires, nov, 2018.

de Sousa Santos, B. Descolonizar el saber, reinventar el poder. Montevideo: Trilce Eds, 2010.

Glowinski, D.; Coll, S. Y.; Baron, N.; Sanchez, M.; Schaerlaeken, S.; Grandjean, D. M. Body, Space, and Emotion. A Perceptual Study. Human technology. 13(1), 32-57, 2017.

Leff, E. Pensamiento ambiental latinoamericano: patrimonio de un saber para la sustentabilidad. In: Pengue, W. (Org.). El pensamiento ambiental del sur. Complejidad, recursos y ecología política latinoamericana. Buenos Aires: Editorial UNGS, 2017.
Llort i Juncadella, I. Ecofeminismo(s) o feminismo ecologista. Estado del debate en la India. Equipo Asia Participación, (1), 1994. Disponible en: https://ecopolitica.org/ ecofeminismos-o-feminismo-ecologista-estado-del-debate-en-la-india/

McDonald, I. Hindu Nationalism, Cultural Spaces, and Bodily Practices in India. American Behavioral Scientist, 46(11), 1563-1576, 2003.

Machado-Aráoz, H. La 'Naturaleza' como objeto colonial. Una mirada desde la condición eco-bio-política del colonialismo contemporáneo. Boletín Onteaikén, 10, 35-47, 2010.

Morley, J. Embodied Consciousness In: Tantric Yoga And The Phenomenology Of Merleau-Ponty. Traversing the Heart. Journeys of the Inter-religious Imagination, 147166, 2010.

Morley, J. Inspiration and Expiration: Yoga Practice through Merleau-Ponty's Phenomenology of the Body. Philosophy East and West, 51(1), 73-82, 2001.

Najmanovich, D. El desafío de la Complejidad: Redes, cartografías dinámicas y mundos implicados. Utopía y Praxis Latinoamericana, 12(38), 71-82, 2007.

Paterson, M. Haptic geographies: ethnography, haptic knowledges and sensuous dispositions. Progress in Human Geography, 33(6), 766-788, 2009.

Pink, S. From embodiment to emplacement: re-thinking competing bodies, senses and spatialities. Sport, Education and Society, 16(3), 343-355, 2011. doi: $10.1080 / 13573322.2011 .565965$

Rao, R. Perception, Cognition and Consciousness in Classical Hindu Psychology. Journal of Consciousness Studies, 12(3), 3-30, 2005.

Rao, R.; Jhansi Rani, N. Body Awareness and Yoga Training. En Perceptual and motor skills, 79(3), 1103-1106, 1994. doi: 10.2466/pms.1994.79.3.1103

Scribano, A. Vigotsky, Bhaskar y Thom: Huellas para la comprensión (y fundamentación) de las Unidades de Experienciación. Revista Latinoamericana de Metodología de la Investigación Social, 1(1), 21-35, 2011.

Scribano, A. Sociología del cuerpo/emoción. RELACES. Revista Latinoamericana de Estudios sobre Cuerpos, Emociones y Sociedad, 4(10), 93-113, 2013. 
Smith, B. R. Body, Mind and Spirit? Towards an Analysis of the Practice of Yoga. Body and Society, 13(2), 25-46, 2007.

Thompson, E. Dreamless Sleep, the Embodied Mind, and Consciousness - The Relevance of a Classical Indian Debate to Cognitive Science. In: Metzinger, T.; Windt, J. M. (Eds.). Open MIND: 37(T). Frankfurt am Main: MIND Group, 2015.
Varela, F.; Maturana, H. El árbol del conocimiento: las bases biológicas del entendimiento humano. Buenos Aires: Lumen, 2003.

Wilber, K. Cuestiones cuánticas. Buenos Aires: Kairós, 1986.

Yi-Fu Tuan. Topofilia. Un estudio sobre percepciones, actitudes y valores medioambientales. España: Melusina, 2007. 